\title{
A METHOD FOR ESTIMATING A PROBABILISTIC DESIGN FACTOR
}

\author{
Edward. E. Osakue', Lucky Anetor ${ }^{2}$ \\ ${ }^{I}$ Department of Industrial Technology, Texas Southern University, Houston, Texas, USA \\ ${ }^{2}$ Department of Mechanical Engineering, Nigerian Defence Academy, Kaduna, Nigeria
}

\begin{abstract}
A probabilistic method for determining a design factor is presented based on the lognormal probability density function. Design parameters are characterized by mean values and coefficients of variation (covs). The design capacity model variability is estimated using first order Taylor series sensitivity analysis. The method provides a lower bound and an upper bound estimate for a design factor which can be used to initiate a design task without an arbitrarily chosen "safety factor". Two design cases of static bending and axial tensile fatigue are considered. In first case, the range of the design factor is 1.485 to 1.620 at a reliability level of $99.9 \%$. In the second case, the range of the design factor is 1.631 to 1.739 at a reliability level of $99 \%$. The higher values of the design factor in the second case compared to the first, even at lower reliability level are attributable to the greater variability of design parameters for the second case. The illustrated two cases are stress-based problems but the method is not limited to stress-based design. It can be applied to other serviceability criteria such as buckling, lateral deflection, torsional deformation, critical frequency, etc. What is required is the formulation of a design capacity model for a failure mode that is related to an appropriate serviceability requirement. Because a probabilistic design factor serves the function of a "safety factor" in deterministic design, it follows that deterministic design is converted to probabilistic design by its use. This is an easier, faster and less costly approach to probabilistic design than classical methods.
\end{abstract}

Keywords: Lognormal, Reliability, Variability, Normal Variate, Design Factor, Over-design Factor

\section{INTRODUCTION}

Engineers and designers have a long history of using "factor of safety", "safety factor", "design factor", "safety margin", etc. in the design of products and components to guard against design uncertainty in order to prevent failure [1- 5]. A safety factor is subjectively assigned in practice so this approach does not provide a logical basis for addressing uncertainty or variability and is not directly related to failure probability. In fact, the safety factor method does not give insight about individual variation or the actual margin of safety in a design [6] because the level of reliability cannot be assessed quantitatively. Hence this approach can lead to over-design and under-design issues. Other terms like reliability factor or reliability index essentially serve the same purpose but are evaluated on the basis of probabilistic and statistical considerations [7, 8, 9]. Probabilistic design treats design model parameters as random variables and allows an assessment of risk or confidence in a design. A reliability factor or index can be quantitatively associated with probability of failure so that safety concerns can be addressed. Probabilistic design can also quantify the amount of over- or under-design [10].

The main factors that affect uncertainties in design are variation in service load, material properties, component geometry, accuracy of analytical model, accuracy of failure mode model, and human errors [11]. Variability in service load is usually the largest but most difficult to predict, especially at the design phase $[5,7,12]$. Variation in material properties and component geometry are controlled by manufacturing practices. For example, tolerances are specified on component dimensions and products to restrict variability and limits are required on material property values for conformance to standards or specifications. Variability in material properties are more significant than those of dimensions which are generally small, especially in mating components. The possibility of human errors is ever present in human activities and design is no exception. Human errors can be greatly minimized by doing things systematically and with a serious commitment to excellence. It is not physically possible or financially feasible to eliminate variation of design parameters. This is due to the fact that the reduction of variability is associated with higher costs either through better and more precise manufacturing methods and processes or increased efforts in quality control. Accepting variability and limiting it seems to be a more pragmatic approach in design as it makes production more costeffective and products more affordable [13].

Due to known variation in many design model parameters, a statistical consideration of their values is a logical expectation in engineering design. This is because statistical methods allow quantitative descriptions of phenomena that show consistent pattern of variability [1]. The need to assess failure risk quantitatively brings probabilistic considerations into design analysis. Different probability distribution functions have been used in describing design parameters. The exponential, normal and lognormal distributions have been used to study reliability and they provide closed form solutions [14, 15, 16, 17]. Reshetov et al [17] has pointed out that the lognormal distribution is more accurate in situations of high reliability than the normal distribution. It is known that products of variates from any distribution tend asymptotically to lognormal [1]. In addition; products, quotients, and exponents of lognormal variates are also 
lognormals. Since design capacity models generally contain products and quotients of design parameters and or sum of terms of products and quotients of design parameters, the lognormal distribution is a good candidate for probabilistic design applications for closed form solutions. Assuming lognormal distribution function for design parameters is reasonable because of the control of material properties and the positive skewness of known load distribution curves [18].

The use of probabilistic and statistical approaches in design analysis has led to the concept of a reliability factor [3, 7]. A reliability factor is expressed as a function of design parameter variability and a failure level variable which represents acceptable safety and or economic risk. Evaluating a reliability factor accurately at the beginning of a design task is problematic in most cases. In general it can only be evaluated approximately, so the approximate value of the reliability factor is here referred to as "design factor". The estimation and use of the design factor will allow design to proceed without an arbitrarily chosen "safety factor". Also a reasonably accurate design factor can minimize the number of iterations for an acceptable design solution.

The objective of this study is to formulate a probabilistic approach for the estimation of design factor. The design parameters of capacity models are assumed to have lognormal probability density function. Each design parameter is characterized by a mean value and a coefficient of variation (cov) and the cov represents the uncertainty associated with the design parameter. The uncertainty of a design capacity model is estimated using sensitivity analysis of the first order Taylor's series expansion. Safety may be quantitatively described in terms of a reliability level denoted by a unit normal variate. The design factor is estimated from a target reliability level and the coefficient of variations (covs) of the design parameters.

\section{DESIGN CAPACITY MODEL}

Random variable analysis shows that mean values of functional relationships are obtained by substituting the mean values of variates [1]. When design capacity models are evaluated using mean values of the significant parameters, the expected result has a reliability of $50 \%$, which corresponds to a unit normal variate of zero. Increased reliability needs higher values of the unit normal variate.

Consider a design capacity model of the form:

$$
X_{M}=f\left(x_{1}, x_{2}, x_{3}, \ldots\right)
$$

Then:

$$
\mu_{m o}=f\left(\mu_{x 1}, \mu_{x 2}, \mu_{x 3}, \ldots\right)
$$

And

$$
s_{m o}=\sqrt{\sum_{i}^{n}\left(\frac{\partial X_{M}}{\partial x_{i}}\right)^{2} s_{x i}^{2}}
$$

The design capacity model basic cov is:

$$
\vartheta_{m o}=\frac{s_{m o}}{\mu_{m o}}=f\left(\vartheta_{x 1}, \vartheta_{x 2}, \vartheta_{x 3}, \ldots\right)
$$

The covs of the above equation come from the load and geometric parameters in a specific design capacity model. The load is by far the most uncertain design parameter [5]. If the load is specified as a maximum value, then a decision need to be made about how much deviation around the maximum value can be expected. For instance does one expect $\pm 5 \%$ or $\pm 10 \%$ deviation on the maximum load value? When the data size is small, or unknown, dividing the deviation by 2 may yield a conservative estimate of the load cov. Another approach for estimating the load cov is the use of overload factors. If an overload factor for a class of devices is given or known over a range [19], both its mean and deviation can be estimated, so the cov can then be determined too. Ullman [20] has suggested the Program Evaluation and Review Technique (PERT) approach for estimating the mean and cov values of service loads. Variations in geometry are controlled by manufacturing practices and these are generally small, especially in mating components which is of the order of 0.001 [1] for crosssectional dimension in machine design. A cov value of 0.005 to 0.03 for length may be assumed in most cases, the higher values being for smaller length sizes.

The basic design capacity model of Eq. (2) needs to be adjusted for analytical accuracy, failure mode correlation to mechanical capability, and human related variability. This could be done by use of coefficients as suggested by [20]. That is:

$$
\mu_{M}=k_{m a} k_{m f} k_{m h} \mu_{m o}
$$

The design capacity model cov is:

$$
\vartheta_{M}^{2}=\vartheta_{m s}^{2}+\vartheta_{m o}^{2}
$$

where:

$$
\vartheta_{m s}^{2}=\vartheta_{m a}^{2}+\vartheta_{m f}^{2}+\vartheta_{m h}^{2}
$$

Engineering design models are approximations of reality and are generally sufficiently accurate, having deviations of about $\pm 10 \%$ [21], representing a $\operatorname{cov}\left(\vartheta_{m a}\right)$ of about $5 \%$. The cov ( $\vartheta_{m f}$ ) of failure mode models vary over a considerable range of 0.02 to $0.25[11,20]$, the higher values being associated with fatigue failures. Human related variability in design can 
be attributed to human errors, most of which may be eliminated by doing things systematically. Human errors can be made during design, manufacturing, assembling, inspection, installation, operation, maintenance, and handling. A study of 931 gear failures over a period of 35 years found that human errors accounted for $40.6 \%$ of the gear failures $[22,23]$. This represents a $\operatorname{cov}\left(\vartheta_{m h}\right)$ of about $7 \%$, assuming the contribution spans 6 standard deviations, since sample size is high. Because gear design is complex, this cov should be conservative for less complicated design tasks.

\section{MECHANICAL CAPABILITY MODEL}

Every component in an assembly or sub-assembly has a limit in its ability to resist imposed load. This limit is here called "mechanical capability" which is the maximum load capacity of the component. A mechanical capability may be the yield strength, tensile strength, fatigue strength, critical lateral deflection, critical torsional deformation, critical axial deformation, critical frequency, etc. of a component. In most cases, mechanical capabilities are determined in controlled environments such as laboratories. Values so obtained are called basic or nominal mechanical capabilities. A component in a device in the field will hardly ever have conditions similar, much less identical, to those in laboratories. Consequently, the basic mechanical capability of a component needs to be adjusted for service conditions. Therefore, the mechanical capability may be expressed as:

$$
\mu_{C}=k_{c r} k_{c t} Y_{c o}
$$

And:

$$
\vartheta_{C}^{2}=\vartheta_{c r}^{2}+\vartheta_{c t}^{2}+\vartheta_{c o}^{2}
$$

Eq. (9) makes correction for reliability level and service temperature. The reliability level correction may be necessary for yield and tensile strengths because these are often quoted as minimum strengths in literature currently. Most fatigue and endurance strengths are reported as mean values [24] so no correction is needed in such cases; that is $k_{c r}=1.0$ and $\vartheta_{c r}=0$. Be sure to confirm if this is true when reading or using data from design handbooks and other reference sources. Sometimes components are deployed in areas of elevated temperatures and adjustment must be made accordingly on the material capability. However, it is preferable to obtain data at service temperatures, especially when safety is a concern. According to Ullman [20], if the material properties are well known, use a $\operatorname{cov}\left(\vartheta_{c o}\right)$ of 0.05 ; if the material properties are not well known, use a cov of $0.10-0.15$. The cov values for fatigue strength from some data indicate it is less than 0.08 [24]. A temperature cov $\left(\vartheta_{c t}\right.$ ) of 0.099 to 0.11 is reported by Budynas and Nissbett [24]. For steel materials, temperature effect may be ignored for temperatures less than $250^{\circ} \mathrm{C}$.
In most fatigue related designs, adjustment is made for surface finish and component size due to their influence on fatigue strength. The cov for the size adjustment is negligible [1] but the cov for the surface finish adjustment factor must be accounted for, so the stress concentration cov is:

$$
\vartheta_{k f}^{2}=\vartheta_{k \sigma}^{2}+\vartheta_{s r}^{2}
$$

The $\operatorname{cov}\left(\vartheta_{k \sigma}\right)$ value for stress concentration factor is 0.11 , while that for the surface finish $\left(\vartheta_{s r}\right)$ is in the range of 0.06 to 0.15 [1].

\section{LOGNORMAL RELIABILITY MODELS}

Two reliability and design factor models based on the lognormal probability density distribution are considered below. They are the load ratio and absolute value models.

\subsection{Load Ratio Model}

Suppose $X_{M}$ and $Y_{C}$ are the design model and service load capability random variables respectively, the load ratio is the quotient of $Y_{C}$ and $X_{M}$. If it is assumed that $Y_{C}$ and $X_{M}$ have lognormal probability density distributions, the load ratio random variable will have lognormal probability density distribution also [1].

That is:

$$
n=\frac{\ln \left(Y_{C}\right)}{\ln \left(X_{M}\right)}=\ln \left[\frac{Y_{C}}{X_{M}}\right]
$$

Fig 1 shows the lognormal domain and standard normal probability distribution representations of $n$. The natural representation uses $n$ as independent variable while the standard normal representation uses the unit normal variate (z) as independent variable. The failure regions are indicated in the two representations. 


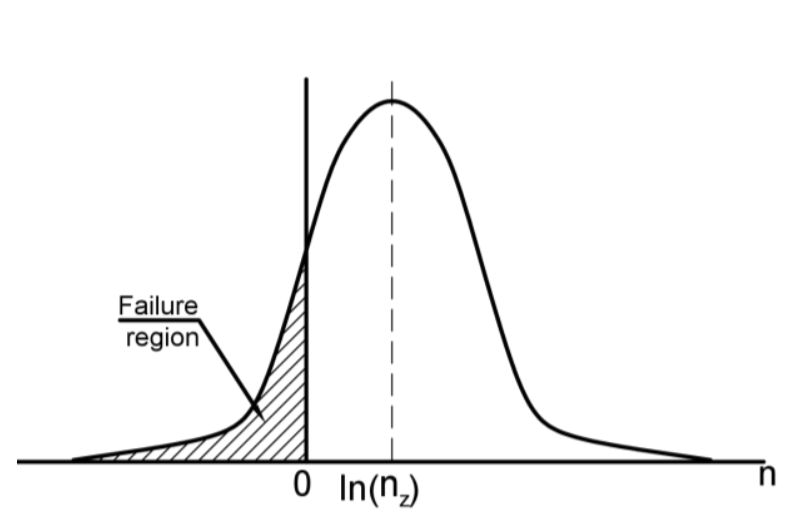

a)

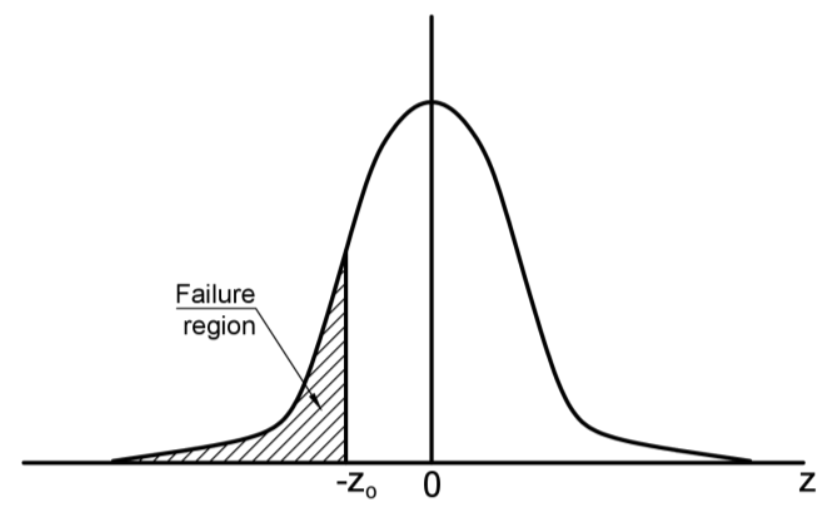

b) Standard normal domain

Fig. 1: Load ratio lognormal probability functions

The mean value of random variable $n$ may be assumed to be equal to the reliability factor $n_{z}[1]$ which is obtained as:

$$
n_{z}=\frac{\mu_{C}}{\mu_{M}}
$$

The reliability factor corresponds to the load ratio of the mean values of the load capability and service load. It is very important to note that the reliability factor depends on the mean values of these design parameters. This is because when considering chance failure, calculations of safety or design factor based on the quotient of minimum load capability and maximum load is not appropriate [1].

The lognormal standard deviation associated with random variable $n$ may be estimated [25] as:

$$
\varpi_{n}=\sqrt{\ln \left[\left(1+\vartheta_{C}^{2}\right)\left(1+\vartheta_{M}^{2}\right)\right]}
$$

The reliability factor is also related to model variability and desired reliability level specified by normal variate $(z)$ [25] as:

$$
n_{z}=\exp \left[\varpi_{n}\left(z+0.5 \varpi_{n}\right)\right]
$$

In design sizing calculations, simplifying assumptions are made to help initiate the normal iterative design process. Generally, design sizing capacity model should be based on the most significant failure mode of a component in order to obtain a reasonable initial size [26]. A reasonable estimate of size in design sizing task should result in fewer iterations during design verification [27]. When a capacity model is based on the most significant failure mode, a less accurate form of Eq. (1) is obtained and may be used for a quick solution. A simplified capacity model can also be obtained by using a linear form of a non-linear model. For instance, Osakue [28] developed a linearized version of the Gerber fatigue model for design sizing but uses the non-linear original model for design verification. Using a simplified form of a design capacity model implies that any estimate of $\vartheta_{M}$ based on such a model is only an approximation. A problem in fatigue situation is that the stress concentration factor depends on component size and the fillet radius. Since the component size and fillet radius are unknown at the beginning of a design task, some assumptions may have to be made. Note that fatigue is reported to be responsible for $80-$ $90 \%$ failures of mechanical devices $[17,29]$. Consequently it may not always be possible at the start of a design endeavor to have an accurate value of $\vartheta_{M}$. Though Eq. (8) is often less complicated than Eq. (1), the above argument may be made for the mechanical capability. In view of these situations, only an approximate value of the reliability factor can be estimated at the beginning of a design task in most cases. This approximate value is called "probabilistic design factor" or simply "design factor" and it is obtained as:

$$
n_{o}=\exp \left[\varpi_{o}\left(z_{o}+0.5 \varpi_{o}\right)\right]
$$

where:

$$
\varpi_{o}=\sqrt{\ln \left[\left(1+\vartheta_{C}^{2}\right)\left(1+\vartheta_{M}^{2}\right)\right]}
$$

The design factor obtained from Eq. (15) is the minimum required value of the design factor for design sizing to avoid under-design condition. Note that Eq. (15) shows the design factor depends only on variability and the desired reliability target.

\subsection{Absolute Value Model}

Fig 2 shows representations of the probability density functions of the design capacity model and component capability model in the physical domain. The covs are shown in brackets. From a probabilistic perspective, failure occurs in the area of overlap between the heel of the design model capacity probability distribution function and the tail of the component capability probability distribution function. 
Theoretically, if the overlapping is prevented, then failure will be eliminated. The gap between the two functions in Fig. 2 is indicated for visualization purposes only. No failure region is indicated because overlapping is assumed not to occur. This is highly unlikely and practically impossible because the probability density distribution functions extend to infinity in both directions. Thus this extreme situation may be associated with over-design, representing an upper bond for the reliability factor. A reliability factor value above this value is too high.

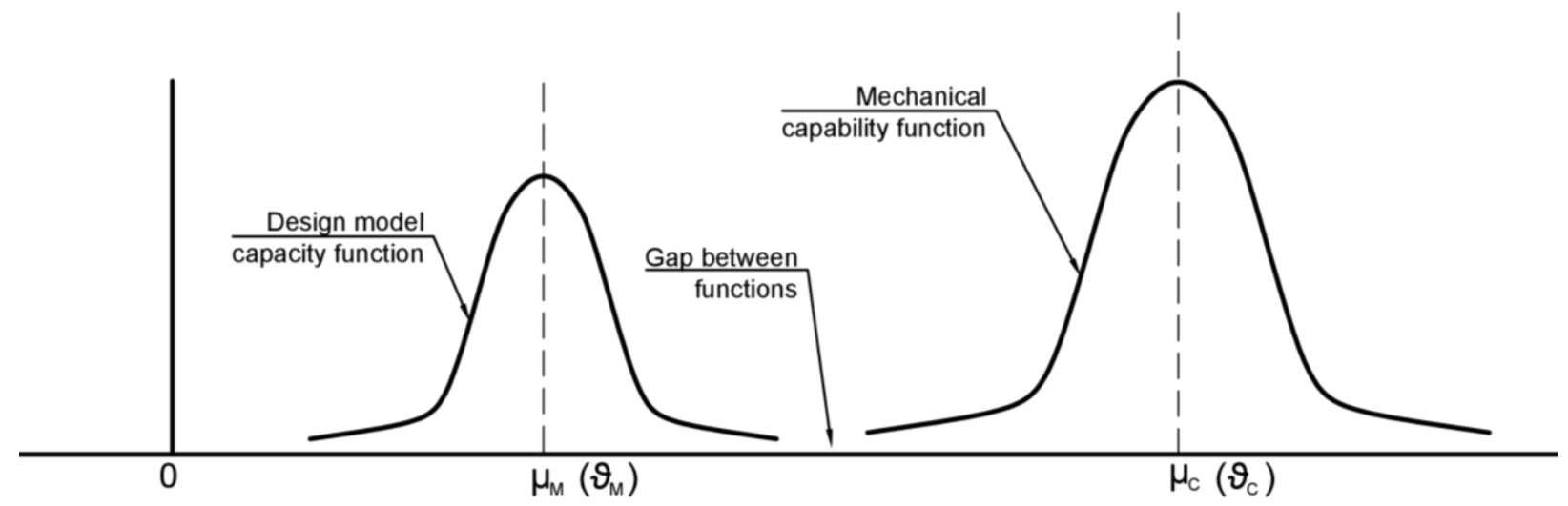

Fig. 2: Physical domain representation of probability density functions

In the lognormal domain:

$$
\ln \left(X_{M}\right)=\ln \left(\mu_{M}\right)-0.5 \varpi_{M}^{2} \pm z \varpi_{M}
$$

And:

$$
\ln \left(Y_{C}\right)=\ln \left(\mu_{C}\right)-0.5 \varpi_{C}^{2} \pm z \varpi_{C}
$$

Where:

$$
\varpi_{M}=\sqrt{\ln \left(1+\vartheta_{M}^{2}\right)}
$$

And:

$$
\varpi_{C}=\sqrt{\ln \left(1+\vartheta_{C}^{2}\right)}
$$

Assuming no overlap of the probability distribution densities of the $X_{M}$ and $Y_{C}$ in the lognormal domain, then:

$$
\begin{aligned}
& \ln \left(\mu_{C}\right)-0.5 \varpi_{C}^{2}-z \varpi_{C} \\
& >\ln \left(\mu_{M}\right)-0.5 \varpi_{M}^{2}+z \varpi_{M}
\end{aligned}
$$

and:

$$
n_{z}^{\prime}>\exp \left[\varpi_{s} z+0.5 \varpi_{a}^{2}\right]
$$

where:

$$
\varpi_{s}=\sqrt{\ln \left(1+\vartheta_{C}^{2}\right)}+\sqrt{\ln \left(1+\vartheta_{M}^{2}\right)}
$$

And

$$
\varpi_{a}^{2}=\ln \left(1+\vartheta_{C}^{2}\right)-\ln \left(1+\vartheta_{M}^{2}\right)
$$

Due to the approximations in the design capacity and component capability models at the beginning of a design task:

$$
n_{o}^{\prime}=\exp \left[\varpi_{s} z_{o}+0.5 \varpi_{a}^{2}\right]
$$

The design factor obtained from Eq. (23) is the maximum value. It is useful in preventing starting a design with too high value of the design factor. During design verification, it should be recalculated and used to judge over-design condition. The objective in design verification is to assess the adequacy of a design. Based on the result(s) of design sizing, the designer(s) have size(s) which can be used to estimate stresses or other performance capacities. The performance capacity result(s) can then be judged for acceptability. If the evaluated design factor for a failure model is higher than the over-design factor $\left(n_{o}^{\prime}\right)$, then reducing the size of the component should be given a serious consideration.

\section{APPLICATIONS OF MODELS}

Two illustrative cases are considered below in demonstrations of model applications. The first case is that of static bending of a flange in a pressure vessel. The second case is the axial tensile loading of a fastener in fatigue. Approximate design sizing capacity models are used to determine the design factors. 


\subsection{Case 1}

Determine the design factor for the thickness of raised face flange of Fig. 3 if the desired reliability is $99.9 \%$ or $z_{o}=3.09$. Yield failure in bending of the flange is most likely and the applied static load has a cov of 0.092 . The outside flange diameter is $1200(1,0.005) \mathrm{mm}$; base flange diameter is $1000(1,0.005) \mathrm{mm}$; and the yield strength of flange has a cov of 0.039 [30]. The mean values of design parameters are outside the parentheses and the covs are the second decimal numbers inside the parentheses above.

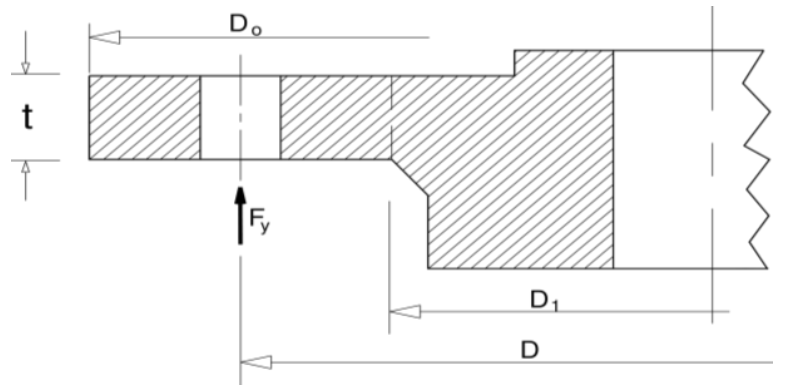

Fig. 3: Flange (After Zhang et al, (2005))

\subsection{Solution for Case 1}

The failure of the flange is critical in bending though direct shear stress is another possible failure model. Neglecting the direct shear stress, the stress capacity model and static yield failure criterion is [30]:

$$
\begin{aligned}
& \sigma_{b z}=\frac{6 F_{y} b}{\pi D_{1} t^{2}} \leq \frac{S_{y t}}{n_{o}} \\
& b=0.5\left(D-D_{1}\right)=0.5 D(1-\beta)
\end{aligned}
$$

Where:

$$
\beta=\frac{D_{1}}{D}
$$

Apply the rule of Eq. (3) to Eqs. (24a to 24c) and obtain:

$$
\vartheta_{m o}^{2}=\vartheta_{F}^{2}+\vartheta_{D 1}^{2}+4 \vartheta_{t}^{2}+\frac{\vartheta_{D}^{2}+\beta^{2} \vartheta_{D 1}^{2}}{(1-\beta)^{2}}
$$

The $\operatorname{cov}\left(\vartheta_{F}\right)$ for the load is $9.2 \%$ and that for flange diameter $\left(\vartheta_{D}\right.$ or $\vartheta_{D 1}$ ) is $0.5 \%$ and that of the thickness ( $\vartheta_{t}$ ) as $0.2 \%$. Substituting values in Eq. (25):

$$
\begin{aligned}
& \vartheta_{m o}^{2}=0.092^{2}+0.005^{2}+4 \times 0.002^{2} \\
& +\frac{0.005^{2}+(1000 / 1200)^{2} 0.005^{2}}{(1-1000 / 1200)^{2}} \\
& =0.01002
\end{aligned}
$$

The model accuracy cov $\left(\vartheta_{m a}\right)$ is taken as $5 \%$ for bending stress formula and the bending failure $\operatorname{cov}\left(\vartheta_{m f}\right)$ is taken as $3 \%$ since the failure mode is well understood. The human errors cov $\left(\vartheta_{m h}\right)$ is assumed to be $3 \%$ because this is a simple design task. Substituting values in Eq. (7):

$$
\vartheta_{m s}^{2}=0.03^{2}+0.05^{2}+0.03^{2}=0.0043
$$

Substituting values of $\vartheta_{m o}$ and $\vartheta_{m s}$ in Eq. (6), the design capacity model cov is:

$$
\vartheta_{M}^{2}=0.0043+0.01002=0.014318
$$

The mechanical load capacity is the yield strength and it has a cov of 0.039 . The yield strength is a mean value, so no reliability level correction is required. Assuming ordinary temperature environment, no service temperature correction is required too, so Eq. (9) becomes:

$$
\vartheta_{C}=\vartheta_{y t}=0.039 ; \quad \vartheta_{C}^{2}=0.00152
$$

\section{Load Ratio Model Solution}

Substituting values of $\vartheta_{M}$ and $\vartheta_{C}$ in Eq. (16):

$$
\begin{aligned}
\varpi_{o}= & \sqrt{\ln [(1+0.00152)(1+0.014318)]} \\
= & 0.12544
\end{aligned}
$$

Substituting values of $\varpi_{o}$ and $z_{o}$ in Eq. (15):

$$
\begin{aligned}
& n_{o}=\exp [0.12544(3.09+0.5 \times 0.12544)] \\
& \quad=1.485
\end{aligned}
$$

\section{Absolute Value Model Solution}

Substituting values of $\vartheta_{M}$ and $\vartheta_{C}$ in Eq. (22a):

$$
\begin{aligned}
\varpi_{s}= & \sqrt{\ln (1+0.00152)} \\
& +\sqrt{\ln (1+0.014318)}=0.1582
\end{aligned}
$$

Substituting values of $\vartheta_{M}$ and $\vartheta_{C}$ in Eq. (22b):

$$
\begin{aligned}
\varpi_{a}^{2}= & \ln (1+0.00152) \\
& -\ln (1+0.014318) \\
& =-0.0127
\end{aligned}
$$


Substituting values of $\varpi_{s}, \varpi_{a}$ and $z_{o}$ in Eq. (23):

$$
\begin{aligned}
n_{o}^{\prime}= & \exp [0.1582 \times 3.09+0.5 \times(-0.0127)] \\
& =1.620
\end{aligned}
$$

\subsection{Case 2}

Two bolts are used to support an external tensile load of 10 $\mathrm{kN}$. For a preliminary design, ISO class 4.6 bolts are specified [19, p. 501 - 502] and preload tension will be applied using a wrench. Estimate the design factor for a reliability of $99 \%$ or $z_{o}=2.326$.

\subsubsection{Solution for Case 2}

Fig. 4 shows a bolt fastener under load in service. The operating forces are the preload tension $\left(F_{i}\right)$ and the external load $\left(F_{e}\right)$. The load on the fastener and treads come from both $F_{e}$ and $F_{i}$. The joint may be assumed to be under fatigue loading due to possible fluctuations in service load and machinery vibrations.

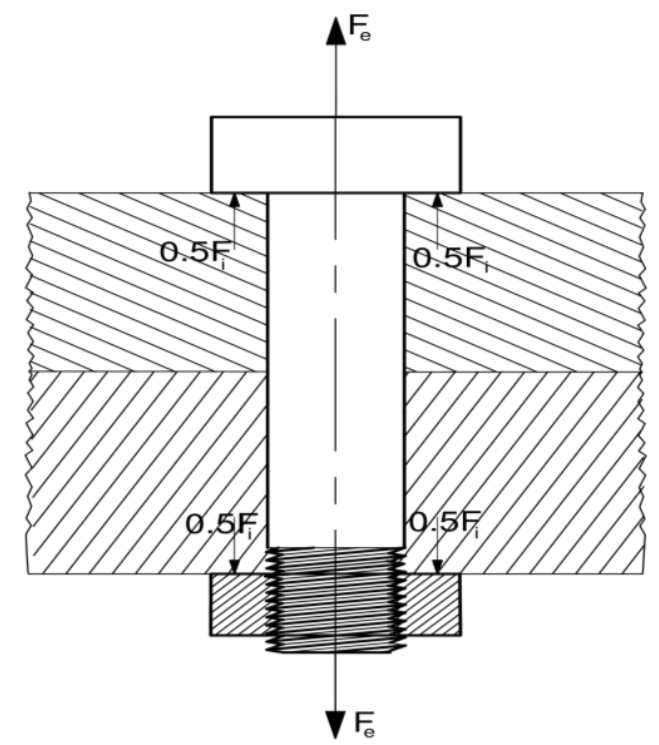

Fig. 4: Fastener under load in service

If a joint is overloaded, then bodily separation of joint parts may occur and the external load is then carried only by the fastener. On this premise, Osakue and Anetor [31] proposed a simple axial fatigue tensile stress capacity model for fastener sizing purposes. This model may be slightly modified by introducing the design factor as:

$$
\sigma_{t}=\frac{k_{\sigma} F_{e}}{A_{t}} \leq \frac{S_{y t}}{n_{o}}
$$

The model capacity basic cov is obtained as:

$$
\vartheta_{m o}^{2}=\vartheta_{F}^{2}+\vartheta_{k f}^{2}+4 \vartheta_{h}^{2}
$$

Hence from Eq. (10) and Eq, (27):

$$
\vartheta_{m o}^{2}=\vartheta_{F}^{2}+\vartheta_{k \sigma}^{2}+\vartheta_{s r}^{2}+4 \vartheta_{h}^{2}
$$

Both the preload and external load are subject to variability, but the preload will in most cases be associated with greater uncertainty because variations of $\pm 25 \%$ in preload torque are routinely experienced [32, 33]. It is therefore more reasonably to use the cov of the preload to estimate the design factor The preload tension is directly proportional to the torque, so from the deviation on the preload torque, the $\operatorname{cov}\left(\vartheta_{F}\right)$ for the load is estimated as $12.5 \%(0.125)$, assuming the deviation spans two standard deviations. It is usually safe to assume that threads have been rolled, except otherwise specified [12]. If the stress concentration type at the thread root is considered as a shoulder type and rolled, the cov for the stress concentration factor $\left(\vartheta_{k \sigma}\right)$ and surface finish $\left(\vartheta_{s r}\right)$ are respectively, 0.11 and 0.06 [1]. The cov for fastener diameter $\left(\vartheta_{h}\right)$ is taken as 0.002. Therefore substituting values in Eq. (28):

$$
\begin{aligned}
\vartheta_{m o}^{2} & =0.125^{2}+0.11^{2}+0.06^{2}+4 \times 0.002^{2} \\
& =0.031341
\end{aligned}
$$

The assembly of fasteners is generally labor intensive so the human error $\operatorname{cov}\left(\vartheta_{m h}\right)$ is taken as 0.05 . The cov for failure model accuracy $\left(\vartheta_{m f}\right)$ for uni-axial fatigue is about 0.05 [20], and the cov for design model accuracy $\left(\vartheta_{m a}\right)$ is taken as 0.05 because it is a simplified model. Substituting values in Eq. (7):

$$
\vartheta_{m s}^{2}=0.05^{2}+0.05^{2}+0.05^{2}=0.0075
$$

Substituting $\vartheta_{m o}$ and $\vartheta_{m s}$ in Eq. (6), the design capacity model cov is:

$$
\begin{aligned}
\vartheta_{M}^{2}= & \vartheta_{m s}^{2}+\vartheta_{m o}^{2}=0.0075+0.031341 \\
& =0.038841
\end{aligned}
$$

The mechanical load capacity is the yield strength and for fastener steel material grades, the properties are well understood. Therefore the cov for the yield strength may be taken as 0.05 [20]. The yield strength for standard fastener is quoted as minimum value [24], so a reliability correction factor is required. Assuming ordinary temperature environment, no service temperature correction is required, so Eq. (9) becomes:

$$
\vartheta_{C}^{2}=\vartheta_{c r}^{2}+\vartheta_{y t}^{2}
$$


From Eq. (A3) in the Appendix:

$$
\vartheta_{c r} \approx z_{s} \vartheta_{y t}^{2}=2.326 \times 0.05^{2}=0.006
$$

Hence from Eq. (29):

$$
\vartheta_{C}^{2}=0.05^{2}+0.006^{2}=0.00254
$$

\section{Load Ratio Model Solution}

Substituting values of $\vartheta_{M}$ and $\vartheta_{C}$ in Eq. (16):

$$
\varpi_{o}=\sqrt{\ln [(1+0.00254)(1+0.038841)]}=0.2016
$$

Substituting values of $\varpi_{o}$ and $z_{o}$ in Eq. (15):

$$
n_{o}=\exp [0.2016(2.326+0.5 \times 0.2016)]=1.631
$$

\section{Absolute Value Model Solution}

Substituting values of $\vartheta_{M}$ and $\vartheta_{C}$ in Eq. (22a):

$$
\begin{aligned}
\varpi_{s}= & \sqrt{\ln (1+0.00254)}+\sqrt{\ln (1+0.038841)} \\
= & 0.2456
\end{aligned}
$$

Substituting values of $\vartheta_{M}$ and $\vartheta_{C}$ in Eq. (22b):

$$
\begin{aligned}
\varpi_{a}^{2}= & \ln (1+0.00254)-\ln (1+0.038841) \\
& =-0.03557
\end{aligned}
$$

Substituting values of $\varpi_{s}, \varpi_{a}$ and $z_{o}$ in Eq. (23):

$$
\begin{aligned}
n_{o}^{\prime}= & \exp [0.2456 \times 2.326+0.5 \times(-0.03557)] \\
& =1.739
\end{aligned}
$$

\section{DISCUSSIONS}

Design capacity model or component capability expressions are of two types; standard and non-standard forms. Eq. 9 is an example of a standard form expression where design parameters are in only multiplicative functional relationship (Eq. 8). Eq. (25) for design Case 1 is an example of nonstandard form expression where design parameter are in multiplicative and or summation functional relationship. The summation relationship of Eq. (25) is in the parameter b, which is given by Eq. (24b). The cov expression for standard form design model capacity can be derived by inspection (please refer to Eqs. (8) and (9)) of the design capacity model functional relationship but the cov expression for non-standard form design model capacity must be derived by use of Eq. (3).
Table 1: Summary Results for Design Cases 1 and 2

\begin{tabular}{|l|l|l|}
\hline Parameter & Case1 & Case2 \\
\hline Reliability target (\%) & 99.9 & 99 \\
\hline Unit normal variate & 3.09 & 2.326 \\
\hline Minimum design factor & 1.485 & 1.631 \\
\hline Maximum design factor & 1.620 & 1.739 \\
\hline
\end{tabular}

Table 1 summarized the results from Case 1 and Case 2 designs. We note that though the reliability in Case 2 is lower than that in Case 1 , the minimum and maximum values of the design factor for Case 2 are higher than those of Case 1. This reason for this is that the covs or variability for the design parameters in Case 2 are higher than those of Case 1. Arbitrarily choosing a "safety factor" may not properly factor this variability information into the value of the design factor. Whether variability is high or low, the risk of failure and its consequence(s) can be properly considered by the choice of a reliability target which is then used to estimate a probabilistic design factor. Consequently, the use of probabilistic design factor makes it possible to fine tune design sizes to the risk of failure.

After initial dimensions are selected, a more accurate capacity model for Case 1 can be developed by considering combined bending and direct shear stresses [26]. Based on such a model, better estimates of the reliability factor and over-design factor can be made. Similarly in Case 2, after design sizing, the non-linear Gerber fatigue design equations $[25,31]$ can be used to obtain estimates of reliability factor and over-design factor.

The above cases demonstrate that probabilistic design allows a quantification of risk which is captured by the design factor. The over-design factor may be interpreted as a value of a design factor for a higher reliability. In that case, it guides the designer in ensuring that sizes are economical. Therefore the over-design factor should be re-calculated during design verification because at design verification it is informative to know if a case of over-design exists. This can be assessed by comparing the estimates of the reliability factor from the design capacity model with that from the absolute value model. If the former is higher than the later, then the component size need to be reduced to prevent overdesign. Consequently, probabilistic design can help to avoid over- or under-design problems while ensuring that safety and quality levels are economically achieved. Over design requires more resources than necessary and leads to costly products. Avoiding over-design helps to conserve product materials and reduce manufacturing resources, machining accuracy, quality control, etc. during processing [34]. Under-design is prevented because the minimum design factor estimate forces the designer to avoid it. Underdesigned products are prone to failures, making the products unsafe and unreliable. This increases the risks of product liability lawsuits, customer dissatisfaction, and even accidents [34]. 


\section{SUMMARY}

A probabilistic method for determining a design factor is presented based on the lognormal probability density function. Design parameters are characterized by mean values and covs. The design capacity model variability is estimated using first order Taylor series sensitivity analysis. The method provides a design factor which can be used to initiate a design task without an arbitrarily chosen "safety factor". The expression for the minimum value of the design factor is based on the load ratio concept, the basis for the standard definition of "factor of safety" or "safety factor". A quantitative expression for over-design is developed and it gives the maximum value for the design factor. The expression is based on the assumption that there is no interaction of the probability density functions of design capacity and component capability models. This situation is very highly unlikely and thus represent an extreme condition.

Two cases of design applications are considered. Case 1 is a static bending stress governed model and the range of the design factor is 1.485 to 1.620 at a reliability level of $99.9 \%$. In Case 2, uni-axial tensile fatigue is assumed and the range of the design factor is 1.631 to 1.739 at a reliability level of $99 \%$. The higher values of Case 2 compared to Case 1 even at lower reliability level are attributable to the greater variability of design parameters for Case 2. The two cases are stress-based design problems. However, the method is not limited to stress-based designs; it can be applied to buckling, deflection, frequency, etc. serviceability criteria. What is required is the formulation of a design capacity model for a failure mode that is related to an appropriate serviceability requirement.

\section{CONCLUSION}

This work demonstrates that a probabilistic design factor can be estimated easily at the beginning of a design task. The factor combines the uncertainties in design and the anticipated failure risks in a single number. A probabilistic design factor is attractive in design works because, it is fineturned to economic and safety risks. Hence it may be argued that what is termed "probabilistic design factor" in this paper is the true "safety factor". Because a probabilistic design factor serves the function of a "safety factor" in deterministic design, it follows that deterministic design is converted to probabilistic design by its use. This is an easier, faster and less costly approach to probabilistic design than classical methods. Generally, probabilistic designs yield smaller sized components that can be produced more economically. Consequently probabilistic design should be encouraged since depletion of scarce resources can be conserved through its use. That is good for the environment and future generations.

Data on mean values and covs of design parameters are required in probabilistic design, so it is suggested that material testing results should preserve such information. Note that the cov is the ratio of the standard deviation to the mean value. While such data may be scarce in public domain, they may well be readily available in private and protected domains.

\section{ACKNOWLEDGEMENT}

The authors gratefully acknowledge that this study was supported in parts with funds from COSET Research Fund and the University Faculty Development Fund of Texas Southern University, Houston, Texas.

\section{NOMENCLATURE}

$A_{t}-$ tensile stress area of fastener

$b$ - flange width

$D_{1}$ - flange base diameter

$D$ - flange outside diameter

$F_{e}$ - external load on fastener

$F_{i}$ - preload tension on fastener

$F_{y}-$ load on flange

$k_{m a}-$ correction multiplier for design capacity model accuracy

$k_{m f}-$ correction multiplier for failure model accuracy

$k_{m h}-$ correction multiplier for human errors

$k_{c r}$ - reliability correction multiplier for basic or nominal capability

$k_{c t}-$ correction multiplier for temperature

$k_{\sigma}-$ stress concentration factor

$n-$ load ratio random variable

$n_{o}-$ design factor

$n_{o}^{\prime}$ - over-design factor

$n_{z}-$ reliability factor

$n_{z}^{\prime}$ - over-design reliability factor

$t$ - flange thickness

$S_{m o}-$ standard deviation of design capacity model

$s_{x i}-$ standard deviation of a design parameter

$S_{y t}$ - mean tensile yield strength

$S_{y t}^{\prime}$ - minimum tensile yield strength

$S_{u t}$ - mean ultimate tensile strength

$S_{u t}^{\prime}-$ minimum ultimate tensile strength

$x_{i}-$ design parameter random variable

$Y_{c o}-$ nominal or basic mechanical capability

$X_{M}$ - design capacity model random variable

$Y_{C}$ - service mechanical capability random variable

$z-$ unit normal variate for a target reliability goal

$z_{o}-$ unit normal variate for target or desired reliability 
$z_{s}-$ unit normal variate for minimum strength

$\beta$ - flange diameter factor

$\frac{\partial}{\partial x}-$ partial differentiation with respect to $x$ variable

$\mu_{m o}-$ mean value of basic design capacity model

$\mu_{C}-$ mean value of service mechanical capability

$\mu_{M}-$ mean value of corrected design capacity model

$\vartheta_{M}-$ effective cov of design capacity model

$\vartheta_{m o}-$ basic cov of design capacity model

$\vartheta_{m a}-$ cov of capacity model accuracy

$\vartheta_{m f}-$ cov of failure mode accuracy

$\vartheta_{m h}-$ human error cov

$\varpi_{o}-$ lognormal standard deviation for design factor

$\varpi_{s}-$ lognormal absolute deviation major factor

$\varpi_{a}-$ lognormal absolute deviation minor factor

$\vartheta_{F}-\operatorname{cov}$ for service load

$\vartheta_{m s}-$ effective miscellaneous cov

$\varpi_{M}-$ lognormal standard deviation for design model capability

$\varpi_{C}-$ lognormal standard deviation for service mechanical capability

$\varpi_{n}-$ lognormal standard deviation for reliability factor

$\vartheta_{D 1}-$ cov for flange base diameter

$\vartheta_{t}-$ cov for flange thickness

$\vartheta_{D}-$ cov for flange outside diameter

$\sigma_{b z}-$ bending stress in flange base

$\sigma_{t}-$ tensile stress in fastener

$\vartheta_{k f}-$ cov of stress concentration

$\vartheta_{k \sigma}-\operatorname{cov}$ for stress concentration factor

$\vartheta_{s r}-\operatorname{cov}$ for stress concentration surface finish modifier

$\vartheta_{y t}-$ cov of tensile yield strength

$\vartheta_{u t}-$ cov of ultimate tensile strength

\section{REFERENCES}

[1] Shigley, E. J. and Mischke, C. R., Standard Handbook of Machine Design, Shigley, J. E. and Mischke, C. R. (Chief Editors), McGraw-Hill, New York, 1996.

[2] Peters, L. C. and Hopkins, B. R., Introduction, Standard Handbook of Machine Design, Shigley, J. E. and Mischke, C. R. (Chief Editors), McGraw-Hill, New York, 1996.
[3] Shigley, E. J. and Mitchell, L. D., Mechanical Engineering Design, McGraw-Hill, New York, 1983.

[4] Johnson, C. R. Optimum Design of Mechanical Elements, Wiley, New York, 1980.

[5] ME 452: Machine Design II, Solution to Homework Set https://www.coursehero.com/file/12146287/hw10solF all2013/

[6] Koch, P., Probabilistic Design: Optimization for SixSigma Quality, 43rd AIAA/ASME/ASCE/AHS/ASC Conference, April 22 - 25. Denver, Colorado, 2002.

[7] Taguchi, G. (Chairman), Taguchi Methods: Research and Development, ASI Dearborn, 1992, pp. 32 - 33, $49-50$.

[8] Mischke, C. R., Statistical Considerations, Standard Handbook of Machine Design, Shigley, J. E. and Mischke, C. R. (Chief Editors), McGraw-Hill, New York, 1996.

[9] Wang, H., Kim, N. H., and Kim, Y., Safety Envelope for Load Tolerance and its Application to Fatigue Reliability, Journal of Mechanical Design, Vol. 128, pp. 919 - 927, 2006.

[10] Cullimore, B., \& Tsuyuki, G., Reliability Engineering and Robust Design: New Methods for Thermal/Fluid Engineering; Proc. of 11th Thermal and Fluid Analysis Workshop, pp. 225 - 240, 2002.

[11] Osakue, E. E., Lognormal Reliability-Based Component Design, Technical report, Department of Industrial Technology, Texas Southern University, Houston, Texas, U.S.A, 2015.

[12] Schmid, S. R., Hamrock, B. J. \& Jacobson,B. O., Fundamentals of Machine Elements, 3rd ed., CRC Press, New York, 2014.

[13] Understanding Probabilistic Design, http://www.kxcad.net/ansys/ANSYS/ansyshelp/Hlp_ GADVPDS1.html,

[14] Shigley, E. J. and Mitchell, L. D., Mechanical Engineering Design, 5th ed., McGraw-Hill, New York, 1989.

[15] Smith, C. O., Introduction to Reliability in Design, McGraw-Hill, New York, Chap. 18, 1976.

[16] O'Connor, P. D. T., Practical Reliability Engineering, Heyden, London, Chap. 4, 1981.

[17] Reshetov, D., Ivanov, A., and Fadeev, V., Reliability of Machines, Moscow, 1980.

[18] JJS, Probabilistic Design, http://telicthoughts.com/probabilistic-design/

[19] Collins, A. J., Busby, H., and Staab, G., Mechanical Design of Machine Elements, John Wiley \& Sons, New Jersey, 2010.

[20] Ullman, G. D, The Mechanical Design Process, 2nd ed., McGraw-Hill, New York, Appendic C.

[21] Matthews, C., ASME Engineer's Data Book, 2nd ed. ASME Press, p. 63, 2005.

[22] Kutz, M., (editor), Mechanical Engineers' Handbook: Materials and Mechanical design, Vol. 1, 3rd ed. John Wiley and Sons, 2006.

[23] Dhillon, B., S., Reliability in the Mechanical Design Process, in Mechanical Engineers' Handbook: 
Materials and Mechanical Design, Vol. 1, 3rd ed. (Edited by Myer Kutz), John Wiley and Sons, New York, 2006.

[24] Budynas, R. G. and Nissbett, J. K., Shigley's Mechanical Engineering Design, 9th ed., McGraw Hill Education.

[25] Osakue, E. E., Probabilistic Design of shaft for Bending and Torsion, International Journal of Research in Engineering and Technology, eISSN: 2319-7308, pp. 370 - 386, 2014.

[26] Osakue, E. E., Anetor, L. and Odetunde, C., Reliability-based Component Design, Proceedings of International Mechanical Engineering Congress and Exposition 2015 MECE, Paper Number IMECE201550700, November 13-19, Houston, Texas, USA, 2015.

[27] Bausbacher, E., Hunt, H., Process Plant Layout and Design, PTR Prentice Hall, Upper Saddle River, New Jersey, 1993.

[28] Osakue, E. E., A Linearized Gerber Fatigue Model, International Journal of Modern Engineering, Vol. 12, No 1, p. $64-72,2012$.

[29] Norton, R. L., Machine Design: An Integrated Approach, Prentice-Hall, Upper Saddle River, New Jersey, 2000.

[30] Zhang, Y. M., He, X. D., Liu, Q. L., Wen, B. C., An Approach of Robust Design for Mechanical Components, Proc. of IMechE, Vol. 219 Part E, pp. $275-283,2005$.

[31] Osakue, E. E. and Anetor, L., Design of Elastic Screw Fasteners under Tensile Load, Mechanical Engineering Research; Vol. 7 No. 1; ISSN 1927-0607 E-ISSN 1927-0615, 2017, doi:10.5539/mer.v7n1pxx URL: $\quad$ https://doi.org/10.5539/mer.v7n1pxx, 2017.

[32] Gope, P. C., Machine Design: Fundamentals and Applications, PHI Learning, Delhi, 2014.

[33] Bolt Tensioning, http://www.lifetimereliability.com/free-articles/precisionmaintenance/Summary_of_Bolt_Tensioning.pdf

[34] Kalpakjian, S. and Schmid, S. R., (2001) Manufacturing Engineering and Technology, 4th Ed. Pearson Prentice Hall, Upper Saddle River, N.J.

\section{APPENDIX: Minimum to Mean Strength Value}

Most data availability on yield and tensile strengths are quoted as minimum values. Assuming lognormal distribution for these mechanical capabilities, the corresponding mean values can be estimated. For case, the mean yield strength is obtained as:

$$
S_{y t}=S_{y t}^{\prime} k_{c r}
$$

Where:

$$
k_{c r}=e^{\vartheta_{y t}\left(z_{s}+0.5 \vartheta_{y t}\right)}
$$

$$
\vartheta_{c r} \approx z_{s} \vartheta_{y t}^{2}
$$

If tensile strength is the serviceability criterion, then:

$$
S_{u t}=S_{u t}^{\prime} k_{c r}
$$

Where:

$$
k_{c r}=e^{\vartheta_{u t}\left(z_{s}+0.5 \vartheta_{u t}\right)}
$$

The cov of the reliability adjustment factor is:

$$
\vartheta_{c r} \approx z_{s} \vartheta_{u t}^{2}
$$

Minimum strength according to ASTM corresponds to $1 \%$ failure level or reliability of $99 \%[1,24]$. A reliability of $99 \%$ corresponds to $z_{s}=2.326$.

The cov of the reliability adjustment factor is: 